COMUNICAÇÃO CIENTÍFICA

\title{
AVALIAÇÃO DO ESTADO NUTRICIONAL DA VIDEIRA 'ITÁLIA' NA REGIÃO DE SÃO MIGUEL ARCANJO-SP, UTILIZANDO O SISTEMA INTEGRADO DE DIAGNOSE E RECOMENDAÇÃ̃O ${ }^{1}$
}

\author{
MAURILO MONTEIRO TERRA ${ }^{2,3}$, IVAN FERDINANDO GERGOLETTI $^{4}$, ERASMO JOSÉ PAIOLI PIRES ${ }^{2,3}$, \\ RENATO VASCONCELOS BOTELHO ${ }^{3,5}$, WAGNER RODRIGUES DOS SANTOS ${ }^{4}$, MARCO ANTONIO TECCHIO $^{2}$
}

RESUMO - Com o objetivo de avaliar o estado nutricional da videira cultivar Itália na região de São Miguel Arcanjo, Estado de São Paulo, Brasil, utilizando o Sistema Integrado de Diagnose e Recomendação (DRIS), foram selecionados 20 vinhedos em produção, com práticas culturais semelhantes. Amostras de limbo e pecíolo foram coletadas em três estádios de desenvolvimento da videira: florescimento, frutos entre ervilha e meia-baga, e início de maturação dos frutos. As amostras de limbo e pecíolo foram analisadas para macronutrientes. A população de referência usada no cálculo das normas DRIS foi à dos vinhedos com produtividade média entre 20,70 e 36,30t/ha. O Índice de Balanço Nutricional (IBN) e a seqüência de deficiência a excesso dos nutrientes foram calculadas para cada vinhedo, nas três épocas de amostragem, para limbo e pecíolo. Nessa avaliação, concluiu-se que o DRIS se mostrou um bom método para avaliação do estado nutricional da videira 'Itália'. De vinte vinhedos avaliados, onze foram considerados em equilíbrio nutricional. Correlações entre IBN e produtividade indicaram que o melhor órgão foi o limbo, e o florescimento a melhor época de amostragem. O DRIS permitiu determinar os vinhedos que, em geral, apresentavam deficiência de fósforo, potássio e magnésio.

Termos para indexação: Vitis, nutrição, macronutrientes, DRIS.

\section{EVALUATION OF THE NUTRITIONAL CONDITION OF ITALIA GRAPEVINE IN THE REGION OF SÃO MIGUEL ARCANJO-SP, USING THE DIAGNOSIS AND RECOMMENDATION INTEGRATED SYSTEM}

\begin{abstract}
This work aimed to evaluate nutritional condition of Italia grapevine in the region centered in São Miguel Arcanjo, SP, Brazil. It was used the Diagnosis and Recommendation Integrated System (DRIS). Twenty bearing vineyards in that region using similar cultural practices were nutritionally surveyed. Leaf blade and petiole samples were taken at three developing phases of grapevine plants: at bloom, in fruits between pea and have-berry and at the beginning of fruit maturation. Leaf blade and petiole samples were analyzed for macronutrients. The reference population used in the calculation of DRIS norms was that of vineyards with average yield between 20.7 to $36.3 \mathrm{t} / \mathrm{ha}$. NBI (Nutritional Balance Index) and the sequence of nutrient limitation were individually calculated for each vineyard at three sample phases and for leaf blade and petiole. In this evaluation permitted to conclude that DRIS was a good method for evaluation of nutritional condition of grapevine cv. Italia. Eleven vineyards were considered nutritionally balanced, with average yield between 20.7 to $36.3 \mathrm{t} / \mathrm{ha}$. Correlations between NBI and yield indicated that leaf blade sampled at bloom was the better choice. The DRIS allowed the determination of vineyards that usually presented a deficiency of phosphorus, potassium and magnesium.
\end{abstract}

Index Terms: Vitis, nutrition, macronutrients, DRIS.

A região de São Miguel Arcanjo, maior produtora de uva Itália do Brasil, possui uma área de $2.282 \mathrm{ha}$, produção de 68.460 toneladas e produtividade média de $30.000 \mathrm{~kg} / \mathrm{ha} \quad$ (Terra et al., 2005). A videira 'Itália' é uma cultivar exigente do ponto de vista nutricional, tanto em qualidade como em quantidade de nutrientes. Por outro lado, a adubação dessa cultivar tem sido feita de forma desequilibrada, gerando desordens nutricionais e auferindo prejuízos ao viticultor devido à queda na produção e na qualidade dos frutos, bem como onerando em demasia a cultura.

Certas plantas, pela sua constituição morfológica, permitem facilmente a separação das folhas em pecíolo e limbo para fins de análise química. O pecíolo do algodoeiro e da videira tem apresentado concentração mais elevada de potássio do que o limbo (Silva et al., 1971; Hiroce \& Terra, 1984; Terra et al., 1998) e, portanto, constitui a parte da folha mais indicada para o estudo

(Trabalho 044-06) Recebido em 17-04-2006. Aceito para publicação em: 29-05-2007.

2 Pesquisadores Científicos do Instituto Agronômico-IAC. Caixa Postal 28. 13001-970. Campinas-SP. Endereços eletrônicos: mmterra@iac.sp.gov.br; ejppires@iac.sp.gov.br; tecchio@iac.sp.gov.br

Bolsitas do CNPq

${ }^{4}$ Bolsistas da FAPESP. Engenheiro Agrônomo e Engenheiro Agrícola. Instituto Agronômico - IAC. Caixa Postal 28. 13001-970. Campinas-SP. mmterra@iac.sp.gov.br

${ }_{5}^{5}$ Professor da Universidade Estadual do Centro Oeste. Rua Simeão Camargo Varela de Sá 03. 85040-080. Guarapuava-PR. rbotelho@unicentro.br 
específico da nutrição potássica. Beattie \& Forshey (1954) utilizaram pecíolo da folha madura mais nova para estudo da situação nutricional de 56 vinhedos da cultivar Concord. Gallo \& Oliveira (1960) utilizaram pecíolo mais limbo da folha madura mais nova da videira cultivar Angélica em diferentes estádios de desenvolvimento da planta. Hiroce \& Terra (1984) determinaram os teores de macronutrientes em pecíolo e limbo, analisados separadamente, da folha recém-madura da videira cultivar Niagara Rosada, no estádio de chumbinho, e concluíram que o pecíolo apresentou concentrações mais elevadas de $\mathrm{K}$ e $\mathrm{Mg}$, e o limbo concentrações mais elevadas de N, Ca e S, sugerindo que se devam levar em conta ambas as partes da folha da videira para estudo da nutrição mineral.

O Sistema Integrado de Diagnose e Recomendação (DRIS) foi originalmente proposto por Beaufils (1973), baseado nas relações de macro e micronutrientes como modelo para identificação de fatores limitantes de produtividade. Entretanto, com o tempo, tem-se mostrado muito mais eficiente como uma forma de interpretação de análise de planta do que como modelo de produtividade agrícola (Bataglia, 1989). Esse método utiliza dados de concentração de nutrientes obtidos por meio da análise de folhas e avalia o estado nutricional da cultura pelas relações de nutrientes, consideradas um parâmetro importante para a cultura da videira, principalmente as relações $\mathrm{K} / \mathrm{Mg}, \mathrm{Ca} / \mathrm{Mg}$ e $\mathrm{Ca} / \mathrm{K}$. Diversos trabalhos identificaram o método DRIS como uma alternativa para avaliar a nutrição das plantas (Beaufils, 1956, para seringueira; Meyer, 1975, para cana-de açúcar; Sumner, 1977, para trigo; Sumner, 1977a, para milho; Sumner, 1978, para soja; Zambello Júnior \& Orlando Filho, 1979, para cana-de-açúcar). No Brasil, Terra et al. (2002) e Terra et al. (2003) avaliaram o estado nutricional da videira 'Itália', respectivamente, nas regiões vitícolas de Jundiaí e Jales, do Estado de São Paulo, usando o DRIS. Ainda, outros trabalhos mostraram vantagens do DRIS em relação ao nível crítico foliar para se fazer diagnose com o propósito de recomendação de complementação de adubação (Escano et al., 1981; Jones, 1981; Jones \& Bowen, 1981; Hanson, 1981; Jones et al., 1986; Walworth \& Sumner, 1987; Schaller, 1988).

A importância do DRIS para a cultura da videira 'Itália' se dá, principalmente, pelo fato de ser uma cultura perene para a qual as desordens nutricionais afetam as plantas cumulativamente ao longo dos anos, além do fato dessas correções de deficiência ou excesso, muitas vezes, não poderem ser feitas durante o ciclo da cultura, tornando a diagnose um fator fundamental no início do mesmo.

Este trabalho teve por objetivo avaliar o estado nutricional da videira 'Itália', em três estádios de desenvolvimento, na região de São Miguel Arcanjo, Estado de São Paulo, Brasil, utilizando o DRIS, por meio da realização de levantamento nutricional em vinhedos produtivos.

Realizou-se levantamento nutricional em vinte propriedades cultivadas com videiras 'Itália' da região de São Miguel Arcanjo, Estado de São Paulo, Brasil, situadas a 2352'S. e $48^{\circ} 00^{\prime} O$., altitude de $650 \mathrm{~m}$ e clima Cfa, segundo Köeppen. A escolha das propriedades foi ao acaso, e de cada propriedade foi selecionado um talhão de vinhedo em produção, com práticas culturais semelhantes. Nos talhões amostrados, havia 230 plantas espaçadas de $4 \mathrm{~m} \times 3 \mathrm{~m}$, com idade média de seis anos e enxertadas sobre o porta-enxerto 420-A. De cada talhão, foram selecionadas 20 plantas ao acaso, das quais foram coletadas cinco folhas por planta, totalizando 100 folhas por amostra. As folhas colhidas foram as recém-maduras de cada ramo produtivo, correspondendo a primeira folha oposta ao $1^{\circ}$ cacho, contado a partir do ápice dos ramos. Das folhas coletadas, separaram-se imediatamente seus pecíolos e limbos, formando amostras distintas. A coleta de folhas foi feita em três épocas distintas: a primeira no florescimento; a segunda quando as bagas estavam no estádio entre ervilha e meia-baga, isto é, fase intermediária, e no início da maturação dos frutos ("veraison") realizou-se a terceira coleta.

As amostras de pecíolos e limbos foram processadas e analisadas separadamente quanto aos teores de $\mathrm{N}, \mathrm{P}, \mathrm{K}, \mathrm{Ca}, \mathrm{Mg}$, $\mathrm{S}, \mathrm{B}, \mathrm{Cu}, \mathrm{Fe}, \mathrm{Mn}$ e $\mathrm{Zn}$, segundo metodologia descrita por Malavolta et al. (1989). Em virtude da contaminação das amostras de folhas por $\mathrm{Cu}, \mathrm{Mn}$ e Zn, principalmente, originária da aplicação de fungicidas contendo esses micronutrientes, foi aplicado o método DRIS somente para os macronutrientes. As concentrações de macronutrientes, no limbo e no pecíolo, em três épocas fenológicas da videira e as produtividades dos vinhedos, foram as variáveis utilizadas no cálculo dos índices DRIS. O estudo do estado nutricional usando o DRIS permitiu, também, determinar a ordem pela qual os macronutrientes estão limitando a produção, mediante os índices DRIS obtidos para cada nutriente.

A interpretação dos resultados de análise foliar, considerando-se a relação de macronutrientes no limbo e no pecíolo e a produção da videira, foi feita aplicando-se o método DRIS. Beaufils (1973), referindo-se ao DRIS, observou que a escolha da população-padrão de referência ou população B é primordial para o sucesso do método e deve constituir-se de plantas não-anormais, isto é, plantas que não tenham sido afetadas por condições anormais de cultivo. O DRIS caracteriza os fatores determinantes da produtividade em termos de índices. Esses fatores podem ser da própria planta, do solo, das práticas culturais e das condições climáticas. Devido o presente trabalho ter sido um estudo do levantamento nutricional utilizando vinhedos sem um maior controle experimental e que refletem com fidelidade as condições produtivas locais, optou-se, na seleção da população $\mathrm{B}$, por vinhedos de média produtividade. Para o estabelecimento das normas necessárias ao cálculo dos índices DRIS, os vinhedos foram separados em duas classes, tomandose como base a produtividade média \pm desvio-padrão $(28,50 \pm$ $7,80 \mathrm{t} / \mathrm{ha}$ ). Os vinhedos com produtividade acima de $36,30 \mathrm{t} / \mathrm{ha} \mathrm{e}$ abaixo de 20,70t/ha foram definidos como população de alta produtividade e população de baixa produtividade, respectivamente, e constituíram a população A. Os demais vinhedos, com média produtividade, constituíram a população $B$ ou de referência. Assim sendo, os vinhedos com produtividades extremas foram excluídos da população de referência. Foram selecionados para os cálculos dos índices DRIS onze vinhedos com produtividade média entre $20,70 \mathrm{t} / \mathrm{ha}$ e $36,30 \mathrm{t} / \mathrm{ha}$. Para as normas, foram calculados a média, o desvio-padrão e o coeficiente de variação, para as relações entre os nutrientes estudados, dois a dois, obtidos da população B. As normas foram assim estabelecidas devido à população $\mathrm{B}$ ser considerada como padrão.

De acordo com esse sistema, também se podem realizar

Rev. Bras. Frutic., Jaboticabal - SP, v. 29, n. 3, p. 710-716, Dezembro 2007 
estudos pelo IBN, que indica equilíbrio ou desequilíbrio nutricional, e corresponde à soma dos valores absolutos dos índices DRIS de cada nutriente dividido pelo número de nutrientes estudados, que, neste trabalho, corresponde a 6 , sendo que, quanto menor o IBN ou mais próximo de zero, melhor o estado nutricional do vinhedo, conforme as observações de Sumner (1977). Os índices DRIS podem variar de valores negativos a positivos, sendo que, em termos de balanço, os índices negativos indicam deficiência dos macronutrientes; quando iguais a zero, indicam que os nutrientes estão em equilíbrio, e quando os índices são positivos, os nutrientes encontram-se em excesso. Dessa maneira, pôde-se determinar a ordem pela qual os macronutrientes estão limitando a produtividade.

Com as normas assim estabelecidas, calcularam-se os índices DRIS e os índices de balanço nutricional (IBN) de acordo com a metodologia desenvolvida por Beaufils (1973) e também relatada por Bataglia \& Dechen (1986), utilizando-se da fórmula:

$$
\text { IndiceX } X=\frac{\left.\left.\sum\left[f\left(\frac{X}{A}\right)\right)_{2} \frac{K}{C V(X / A)}+f\left(\frac{X}{B}\right)\right)_{2} \frac{K}{C V(X / B)}+\cdots+f\left(\frac{X}{Z}\right)_{n} \frac{K}{C V(X / Z)}\right]-\sum\left[f\left(\frac{a}{X}\right) \frac{K}{1 C V(a / X)}+f\left(\frac{b}{X}\right)_{2} \frac{K}{C V(b / X)}+\cdots+f\left(\frac{z}{X}\right)_{n} \frac{K}{C V(z / X)}\right]}{n+m}
$$

$\mathrm{IBN}=\mathrm{S}[$ índice $\mathrm{X}]$ em valores absolutos.

onde:

$A, B, \ldots, Z$ e a, b,..., z- representam qualquer concentração de macronutrientes possíveis de diagnose;

CV- é o coeficiente de variação da relação na população nãoanormal;

$\mathrm{K}$ - é o coeficiente de sensibilidade, de valor arbitrário $=10$;

$\mathrm{X} / \mathrm{A}, \mathrm{X} / \mathrm{B}, \ldots, \mathrm{X} / \mathrm{Z}$ - são as relações dos macronutrientes na amostra; $\mathrm{n}-\mathrm{S} \mathrm{X} / \mathrm{A}+\mathrm{X} / \mathrm{B}+\ldots+\mathrm{X} / \mathrm{Z}, \mathrm{e}$

$\mathrm{m}-\mathrm{Sa} / \mathrm{X}+\mathrm{b} / \mathrm{X}+\ldots+\mathrm{z} / \mathrm{X}$.

Coeficiente negativo de correlação entre IBN e produtividade indica desequilíbrio, ou seja, quanto mais negativa a correlação, menor a produtividade esperada.

Verifica-se, na Tabela 1, que os teores de $\mathrm{Cu}, \mathrm{Mn}$ e $\mathrm{Zn}$, tanto nos pecíolos quanto nos limbos, de folhas coletadas no florescimento e no início da maturação dos frutos, apresentamse com valores excessivos, quando comparados com os limites e as faixas de concentrações propostos por Terra et al. (1998) e Terra (2003) para videiras, devido à contaminação das amostras de folhas por aplicação de fungicidas contendo estes micronutrientes. Assim sendo, foi aplicado o método DRIS somente para os macronutrientes.

De modo geral, a ordem de limitação dos nutrientes de cada vinhedo não foi inteiramente coincidente quanto aos diferentes tecidos dentro de uma mesma época de amostragem. $\mathrm{O}$ mesmo fato foi observado quanto às épocas de amostragem em relação a somente um tecido analisado, demonstrando grande influência do tecido e da época de amostragem dos mesmos na diagnose pelo DRIS (Tabelas 2 a 7).

A soma dos índices DRIS originou outro índice muito importante: o índice de balanço nutricional (IBN). Verifica-se, nas Tabelas 2 a 7, que, no geral, os valores dos índices de balanço nutricional foram mais diferentes comparando-se épocas e tecidos amostrados. Os efeitos da idade e do tecido amostrado, a posição da folha amostrada na planta e a cultivar podem ser minimizados pelo uso do método DRIS, estando de acordo com trabalhos verificados por Beaufils (1973), Sumner \& Beaufils (1975) e Sumner (1977a).

Correlações entre IBN e produtividade, considerando-se os limbos e os pecíolos, e as épocas de coletas, encontram-se na Tabela 8. Foi encontrada correlação negativa para limbo no florescimento, ou seja, $-0,1637$ para macronutrientes. Portanto, o limbo, quando coletado no florescimento, indicou o melhor estado nutricional dos vinhedos. Terra et al. (2002) e Terra et al. (2003), avaliando o estado nutricional da videira 'Itália', nas regiões de Jundiaí e Jales, Estado de São Paulo, Brasil, usando o DRIS, concluíram que o limbo foi o órgão ideal de coleta.A época mais adequada para a amostragem de folhas foi no florescimento, pelo fato de maior coerência dos índices de balanço nutricional adotados como referência. Esses resultados são concordantes com os de Christensen et al. (1982), Terra et al. (2002) e Terra et al. (2003), que afirmaram que a coleta do órgão para amostragem deve ser realizada no florescimento. A partir da definição do melhor órgão e da melhor época de amostragem de folhas, procedeu-se ao estudo de cada vinhedo em particular, considerando-se apenas os dados de limbo coletados no florescimento (Tabela 2). Observou-se que, quando há nutrientes deficientes no balanço nutricional, a ordem de grandeza dos índices é que orientará a escolha dos melhores tratamentos visando ao aumento da produção, o que concorda com as observações de Sumner (1977a). Pela interpretação desses dados, verificaram-se desordens nutricionais ligadas à deficiência de fósforo, potássio e magnésio. As correlações entre IBN e produtividade, e as seqüências de deficiência a excesso de nutrientes demonstraram que os tecidos e os estádios de desenvolvimento da videira 'Itália' influíram na diagnose nutricional, utilizando-se do método DRIS. As seqüências de deficiência a excesso de nutrientes dos vinhedos amostrados dentro de uma mesma época e tecidos não tiveram semelhanças entre si, refletindo as condições locais e o manejo de cada vinhedo.

Os valores dos índices DRIS para os macronutrientes e dos balanços nutricionais, quando iguais a zero, indicam equilíbrio nutricional. Para fins práticos, esses índices necessariamente não precisam ser iguais a zero, mas, sim, estarem próximos dele. Os intervalos dentro dos quais os índices DRIS indicariam um relativo equilíbrio, dependem da experiência do pesquisador. Entretanto, os índices mais negativos e, também, os mais positivos são os que devem ter maior importância na recomendação da adubação. Jones (1981) afirmou que respostas na produtividade podem ser esperadas sempre que o índice DRIS de um nutriente for negativo, sendo que a magnitude da resposta dependerá de outros fatores, como o grau de deficiência do nutriente, presença de outros nutrientes deficientes e estresses ambientais.

O IBN indica o estado nutricional do vinhedo e quanto menor esse índice, melhor o equilíbrio nutricional. Porém, na Tabela 2, observa-se que alguns vinhedos com baixa produtividade apresentaram IBN inferior aos vinhedos da população de referência. Nem sempre quando uma planta está em equilíbrio nutricional adequado, significa que ela terá alta produtividade, sendo apenas o inverso verdadeiro (Snöeck, 1984). O vinhedo 9, constante da Tabela 2, exemplifica bem o fato. Leite 
(1993) observou o mesmo fato avaliando o estado nutricional de cafeeiros, utilizando o DRIS, concluindo que, nesses casos, a principal limitação na produtividade foi de ordem não-nutricional. O reduzido tamanho da população no levantamento nutricional com produtividades altas, médias e baixas, e a seleção da população de referência, como a de produtividade média, não influíram negativamente na utilização do DRIS. Letzsch \& Sumner (1984) sugeriram, para o correto desenvolvimento das normas do DRIS, usar substancial número de indivíduos, com pelo menos $10 \%$ com alta produtividade.

Condições reais sem um maior controle experimental refletindo as condições locais de produção são importantes no levantamento nutricional. O DRIS foi um critério adequado e mostrou-se promissor na diagnose nutricional da videira 'Itália'. Para melhor verificar-se a influência da adubação nos níveis de nutrientes na videira 'Itália' através do DRIS, experimentos mais controlados, como ensaios de adubação, fazem-se necessários. O limbo coletado no estádio de florescimento foi a condição que melhor refletiu o estado nutricional da videira.

O DRIS mostrou-se um bom método para a avaliação do estado nutricional da videira cultivar Itália.

TABELA 1 - Concentração média dos nutrientes nos limbos e pecíolos de uva 'Itália', em função de épocas de amostragem. São Miguel Arcanjo-SP.

\begin{tabular}{|c|c|c|c|c|c|c|c|c|c|c|c|c|}
\hline \multirow{2}{*}{ AMOSTRA } & \multirow{2}{*}{ ÉPOCA } & \multicolumn{11}{|c|}{ NUTRIENTES } \\
\hline & & $\mathbf{N}$ & $\mathbf{P}$ & $\mathbf{K}$ & $\mathbf{C a}$ & Mg & $\mathbf{S}$ & $\mathbf{B}$ & $\mathbf{C u}$ & $\mathbf{F e}$ & Mn & $\mathbf{Z n}$ \\
\hline \multirow{3}{*}{ Limbo } & & & & & $\mathrm{kg} \mathrm{-.}$ & & & & & $----m$ & g --... & \\
\hline & Florescimento & 36 & 3,6 & 13 & 20 & 3,3 & 4,1 & 26 & 116 & 229 & 351 & 134 \\
\hline & $\begin{array}{l}\text { Ervilha/meia- } \\
\text { baga }\end{array}$ & 31 & 2,7 & 29 & 22 & 3,2 & 3,4 & 29 & 101 & 245 & 334 & 126 \\
\hline \multirow{4}{*}{ Pecíolo } & Maturação & 32 & 2,3 & 13 & 28 & 4,1 & 2,8 & 31 & 121 & 259 & 309 & 125 \\
\hline & Florescimento & 14 & 4,3 & 25 & 15 & 4,1 & 1,1 & 21 & 51 & 66 & 299 & 77 \\
\hline & $\begin{array}{l}\text { Ervilha/meia- } \\
\text { baga }\end{array}$ & 9,4 & 3,7 & 62 & 14 & 5,7 & 0,7 & 30 & 57 & 60 & 282 & 87 \\
\hline & Maturação & 11 & 3,6 & 43 & 21 & 9,1 & 1,2 & 36 & 54 & 62 & 283 & 90 \\
\hline
\end{tabular}

TABELA 2 - Produtividade, Índices DRIS, Índices de Balanço Nutricional (IBN) e seqüência de deficiência a excesso nutricional para os vinhedos amostrados considerando limbo $-1^{\text {a }}$ coleta (Florescimento). São Miguel Arcanjo-SP.

\begin{tabular}{ccrrrrrrrr}
\hline Vinhedo & $\begin{array}{c}\text { Prod. } \\
\text { t/ha }\end{array}$ & \multicolumn{1}{c}{ Índice DRIS } & IBN & $\begin{array}{c}\text { Seqüência deficiência a } \\
\text { excesso }\end{array}$ \\
\hline \multicolumn{1}{c}{} & $\mathrm{N}$ & $\mathrm{P}$ & $\mathrm{K}$ & $\mathrm{Ca}$ & $\mathrm{Mg}$ & $\mathrm{S}$ & \\
\hline 01 & 30 & 3 & 28 & -38 & 11 & -3 & -1 & 14 & $\mathrm{~K}>\mathrm{Mg}>\mathrm{S}>\mathrm{N}>\mathrm{Ca}>\mathrm{P}$ \\
02 & 27 & -14 & -24 & 9 & -18 & 5 & 43 & 19 & $\mathrm{P}>\mathrm{Ca}>\mathrm{N}>\mathrm{Mg}>\mathrm{K}>\mathrm{S}$ \\
03 & 18 & -26 & -14 & 3 & 21 & 38 & -22 & 21 & $\mathrm{~N}>\mathrm{S}>\mathrm{P}>\mathrm{K}>\mathrm{Ca}>\mathrm{Mg}$ \\
04 & 40 & 7 & -3 & 11 & -8 & -19 & 12 & 10 & $\mathrm{Mg}>\mathrm{Ca}>\mathrm{P}>\mathrm{N}>\mathrm{K}>\mathrm{S}$ \\
05 & 32 & -8 & -10 & 0 & 23 & -1 & -4 & 8 & $\mathrm{P}>\mathrm{N}>\mathrm{S}>\mathrm{Mg}>\mathrm{K}>\mathrm{Ca}$ \\
06 & 41 & 8 & -19 & 37 & 25 & -47 & -4 & 23 & $\mathrm{Mg}>\mathrm{P}>\mathrm{S}>\mathrm{N}>\mathrm{Ca}>\mathrm{K}$ \\
07 & 38 & -5 & -4 & -7 & -11 & -11 & 37 & 12 & $\mathrm{Ca}=\mathrm{Mg}>\mathrm{K}>\mathrm{P}>\mathrm{N}>\mathrm{S}$ \\
08 & 29 & 3 & -16 & 28 & -14 & 8 & -8 & 13 & $\mathrm{P}>\mathrm{C}>\mathrm{S}>\mathrm{N}>\mathrm{Mg}>\mathrm{K}$ \\
09 & 18 & -8 & 8 & 0 & 24 & 3 & -25 & 11 & $\mathrm{~S}>\mathrm{N}>\mathrm{K}>\mathrm{Mg}>\mathrm{P}>\mathrm{Ca}$ \\
10 & 37 & -20 & 2 & 0 & 10 & 25 & 29 & 9 & $\mathrm{~N}>\mathrm{S}>\mathrm{K}>\mathrm{P}>\mathrm{Ca}>\mathrm{Mg}$ \\
11 & 33 & -15 & -50 & -2 & 13 & 21 & 22 & 22 & $\mathrm{P}>\mathrm{N}>\mathrm{K}>\mathrm{Ca}>\mathrm{Mg}>\mathrm{S}$ \\
12 & 18 & -2 & 8 & 23 & 10 & 10 & -48 & 17 & $\mathrm{~S}>\mathrm{N}>\mathrm{P}>\mathrm{Ca}=\mathrm{Mg}>\mathrm{K}$ \\
13 & 27 & 2 & 9 & 17 & 14 & -15 & -27 & 14 & $\mathrm{~S}>\mathrm{Mg}>\mathrm{N}>\mathrm{P}>\mathrm{Ca}>\mathrm{K}$ \\
14 & 25 & 6 & 8 & -9 & 16 & -21 & 1 & 10 & $\mathrm{Mg}>\mathrm{K}>\mathrm{S}>\mathrm{N}>\mathrm{P}>\mathrm{Ca}$ \\
15 & 23 & -11 & -1 & 1 & 13 & 12 & -14 & 9 & $\mathrm{~S}>\mathrm{N}>\mathrm{P}>\mathrm{K}>\mathrm{Mg}>\mathrm{Ca}$ \\
16 & 38 & 4 & -21 & 7 & -19 & 1 & 28 & 13 & $\mathrm{P}>\mathrm{Ca}>\mathrm{Mg}>\mathrm{N}>\mathrm{K}>\mathrm{S}$ \\
17 & 30 & 4 & 15 & -13 & -18 & -2 & 13 & 11 & $\mathrm{Ca}>\mathrm{K}>\mathrm{Mg}>\mathrm{N}>\mathrm{S}>\mathrm{P}$ \\
18 & 21 & 3 & 3 & -11 & -13 & 19 & -2 & 8 & $\mathrm{Ca}>\mathrm{K}>\mathrm{S}>\mathrm{N}=\mathrm{P}>\mathrm{Mg}$ \\
19 & 30 & -2 & -6 & 12 & -23 & -3 & 22 & 11 & $\mathrm{Ca}>\mathrm{P}>\mathrm{Mg}>\mathrm{N}>\mathrm{K}>\mathrm{S}$ \\
20 & 15 & 3 & -31 & 20 & -21 & -19 & 48 & 24 & $\mathrm{P}>\mathrm{Ca}>\mathrm{Mg}>\mathrm{N}>\mathrm{K}>\mathrm{S}$ \\
\hline
\end{tabular}

TABELA 3 - Produtividade, Índices DRIS, Índices de Balanço Nutricional (IBN) e seqüência de deficiência a excesso nutricional para os vinhedos amostrados considerando limbo $-2^{\mathrm{a}}$ coleta (Fruto entre ervilha e meia-baga). São Miguel Arcanjo-SP

\begin{tabular}{|c|c|c|c|c|c|c|c|c|c|}
\hline \multirow[t]{2}{*}{ Vinhedo } & \multirow[t]{2}{*}{$\begin{array}{c}\text { Prod. } \\
\text { t/ha }\end{array}$} & \multicolumn{6}{|c|}{ Índice DRIS } & \multirow[t]{2}{*}{ IBN } & \multirow[t]{2}{*}{$\begin{array}{c}\text { Seqüência deficiência a } \\
\text { excesso }\end{array}$} \\
\hline & & $\mathrm{N}$ & $\mathrm{P}$ & $\mathrm{K}$ & $\mathrm{Ca}$ & $\mathrm{Mg}$ & $\mathrm{S}$ & & \\
\hline 01 & 30 & -12 & 14 & -3 & 4 & -4 & 1 & 6 & $\mathrm{~N}>\mathrm{Mg}>\mathrm{K}>\mathrm{S}>\mathrm{Ca}>\mathrm{P}$ \\
\hline 02 & 27 & -13 & 15 & -2 & -16 & 2 & 14 & 10 & $\mathrm{Ca}>\mathrm{N}>\mathrm{K}>\mathrm{Mg}>\mathrm{S}>\mathrm{P}$ \\
\hline 03 & 18 & -20 & -18 & -1 & 2 & 43 & -5 & 15 & $\mathrm{~N}>\mathrm{P}>\mathrm{S}>\mathrm{K}>\mathrm{Ca}>\mathrm{Mg}$ \\
\hline 04 & 40 & 10 & 0 & -20 & 20 & -18 & 7 & 12 & $\mathrm{~K}>\mathrm{Mg}>\mathrm{P}>\mathrm{S}>\mathrm{N}>\mathrm{Ca}$ \\
\hline 05 & 32 & -8 & -17 & 2 & 30 & -13 & 7 & 13 & $\mathrm{P}>\mathrm{Mg}>\mathrm{N}>\mathrm{K}>\mathrm{S}>\mathrm{Ca}$ \\
\hline 06 & 41 & 5 & -28 & 17 & 32 & -48 & 22 & 25 & $\mathrm{Mg}>\mathrm{P}>\mathrm{N}>\mathrm{K}>\mathrm{S}>\mathrm{Ca}$ \\
\hline 07 & 38 & 10 & -15 & 17 & -13 & -20 & 20 & 16 & $\mathrm{Mg}>\mathrm{P}>\mathrm{Ca}>\mathrm{N}>\mathrm{K}>\mathrm{S}$ \\
\hline 08 & 29 & 12 & -15 & -38 & 17 & 16 & 8 & 18 & $\mathrm{~K}>\mathrm{P}>\mathrm{S}>\mathrm{N}>\mathrm{Mg}>\mathrm{Ca}$ \\
\hline 09 & 18 & 6 & -16 & 26 & 7 & -2 & -22 & 13 & $\mathrm{~S}>\mathrm{P}>\mathrm{Mg}>\mathrm{N}>\mathrm{Ca}>\mathrm{K}$ \\
\hline 10 & 37 & -37 & 12 & -11 & 18 & 13 & 5 & 16 & $\mathrm{~N}>\mathrm{K}>\mathrm{S}>\mathrm{P}>\mathrm{Mg}>\mathrm{Ca}$ \\
\hline 11 & 33 & -5 & -14 & -3 & -5 & 18 & 9 & 9 & $\mathrm{P}>\mathrm{N}=\mathrm{Ca}>\mathrm{K}>\mathrm{S}>\mathrm{Mg}$ \\
\hline 12 & 18 & 5 & -11 & 7 & 6 & 37 & -44 & 18 & $\mathrm{~S}>\mathrm{P}>\mathrm{N}>\mathrm{Ca}>\mathrm{K}>\mathrm{Mg}$ \\
\hline 13 & 27 & -3 & -12 & 12 & 7 & -13 & 10 & 10 & $\mathrm{Mg}>\mathrm{P}>\mathrm{N}>\mathrm{Ca}>\mathrm{S}>\mathrm{K}$ \\
\hline 14 & 25 & -3 & -11 & 15 & 3 & -23 & 19 & 12 & $\mathrm{Mg}>\mathrm{P}>\mathrm{N}>\mathrm{Ca}>\mathrm{K}>\mathrm{S}$ \\
\hline 15 & 23 & -9 & 16 & 9 & -3 & 18 & -31 & 14 & $\mathrm{~S}>\mathrm{N}>\mathrm{Ca}>\mathrm{K}>\mathrm{P}>\mathrm{Mg}$ \\
\hline 16 & 38 & 33 & -15 & -47 & 4 & 34 & -9 & 24 & $\mathrm{~K}>\mathrm{P}>\mathrm{S}>\mathrm{Ca}>\mathrm{N}>\mathrm{Mg}$ \\
\hline 17 & 30 & 15 & 25 & 15 & -23 & -17 & -16 & 18 & $\mathrm{Ca}>\mathrm{Mg}>\mathrm{S}>\mathrm{N}=\mathrm{K}>\mathrm{P}$ \\
\hline 18 & 21 & -5 & -7 & 13 & -10 & 11 & -2 & 8 & $\mathrm{Ca}>\mathrm{P}>\mathrm{N}>\mathrm{S}>\mathrm{Mg}>\mathrm{K}$ \\
\hline 19 & 30 & 12 & -12 & -48 & 10 & 31 & 8 & 20 & $\mathrm{~K}>\mathrm{P}>\mathrm{S}>\mathrm{Ca}>\mathrm{N}>\mathrm{Mg}$ \\
\hline 20 & 15 & 31 & -18 & -31 & 7 & 9 & 1 & 16 & $\mathrm{~K}>\mathrm{P}>\mathrm{S}>\mathrm{Ca}>\mathrm{Mg}>\mathrm{N}$ \\
\hline
\end{tabular}


TABELA 4 - Produtividade, Índices DRIS, Índices de Balanço Nutricional (IBN) e seqüência de deficiência a excesso nutricional para os vinhedos amostrados considerando limbo $-3^{\mathrm{a}}$ coleta (Início de maturação dos frutos). São Miguel Arcanjo-SP.

\begin{tabular}{ccrrrrrrrr}
\hline Vinhedo & $\begin{array}{c}\text { Prod. } \\
\text { t/ha }\end{array}$ & \multicolumn{1}{c}{ Índice DRIS } & IBN & $\begin{array}{c}\text { Seqüência deficiência a } \\
\text { excesso }\end{array}$ \\
\hline \multicolumn{1}{c}{} & $\mathrm{N}$ & $\mathrm{P}$ & $\mathrm{K}$ & $\mathrm{Ca}$ & $\mathrm{Mg}$ & $\mathrm{S}$ & \\
\hline 01 & 30 & -20 & 21 & -22 & 24 & 10 & -13 & 18 & $\mathrm{~K}>\mathrm{N}>\mathrm{S}>\mathrm{Mg}>\mathrm{P}>\mathrm{Ca}$ \\
02 & 27 & -13 & 15 & -5 & -10 & 10 & 3 & 9 & $\mathrm{~N}>\mathrm{Ca}>\mathrm{K}>\mathrm{S}>\mathrm{Mg}>\mathrm{P}$ \\
03 & 18 & -11 & 10 & -6 & -6 & 33 & -19 & 14 & $\mathrm{~S}>\mathrm{N}>\mathrm{K}=\mathrm{Ca}>\mathrm{P}>\mathrm{Mg}$ \\
04 & 40 & -6 & 5 & 19 & 10 & -27 & 0 & 11 & $\mathrm{Mg}>\mathrm{N}>\mathrm{S}>\mathrm{P}>\mathrm{Ca}>\mathrm{K}$ \\
05 & 32 & -7 & -7 & -9 & 27 & -16 & 12 & 13 & $\mathrm{Mg}>\mathrm{K}>\mathrm{N}=\mathrm{P}>\mathrm{S}>\mathrm{Ca}$ \\
06 & 41 & 10 & -19 & -12 & 34 & -49 & 36 & 27 & $\mathrm{Mg}>\mathrm{P}>\mathrm{K}>\mathrm{N}>\mathrm{Ca}>\mathrm{S}$ \\
07 & 38 & 8 & 0 & 12 & -7 & -13 & 1 & 7 & $\mathrm{Mg}>\mathrm{C}>\mathrm{P}>\mathrm{S}>\mathrm{N}>\mathrm{K}$ \\
08 & 29 & -5 & -8 & 0 & 2 & 9 & 3 & 4 & $\mathrm{P}>\mathrm{N}>\mathrm{K}>\mathrm{Ca}>\mathrm{S}>\mathrm{Mg}$ \\
09 & 18 & -6 & -14 & 40 & -3 & 1 & -19 & 14 & $\mathrm{~S}>\mathrm{P}>\mathrm{N}>\mathrm{Ca}>\mathrm{Mg}>\mathrm{K}$ \\
10 & 37 & -15 & 34 & -28 & -2 & -4 & 14 & 16 & $\mathrm{~K}>\mathrm{N}>\mathrm{Mg}>\mathrm{Ca}>\mathrm{S}>\mathrm{P}$ \\
11 & 33 & 7 & -32 & -12 & -5 & 19 & 23 & 16 & $\mathrm{P}>\mathrm{K}>\mathrm{Ca}>\mathrm{N}>\mathrm{Mg}>\mathrm{S}$ \\
12 & 18 & -6 & 4 & -5 & 4 & 42 & -40 & 17 & $\mathrm{~S}>\mathrm{N}>\mathrm{K}>\mathrm{P}=\mathrm{Ca}>\mathrm{Mg}$ \\
13 & 27 & 7 & -12 & -3 & 11 & -13 & 11 & 10 & $\mathrm{Mg}>\mathrm{P}>\mathrm{K}>\mathrm{N}>\mathrm{Ca}=\mathrm{S}$ \\
14 & 25 & 15 & -10 & 4 & 14 & -21 & -2 & 11 & $\mathrm{Mg}>\mathrm{P}>\mathrm{S}>\mathrm{K}>\mathrm{Ca}>\mathrm{N}$ \\
15 & 23 & 19 & -4 & 1 & -2 & 15 & -29 & 12 & $\mathrm{~S}>\mathrm{P}>\mathrm{Ca}>\mathrm{K}>\mathrm{Mg}>\mathrm{N}$ \\
16 & 38 & -5 & -4 & -5 & -11 & 26 & 0 & 8 & $\mathrm{Ca}>\mathrm{N}=\mathrm{K}>\mathrm{P}>\mathrm{S}>\mathrm{Mg}$ \\
17 & 30 & 4 & 3 & 34 & -24 & -21 & 4 & 15 & $\mathrm{Ca}>\mathrm{Mg}>\mathrm{P}>\mathrm{N}=\mathrm{S}>\mathrm{K}$ \\
18 & 21 & -13 & 12 & 12 & -17 & 1 & 5 & 10 & $\mathrm{Ca}>\mathrm{N}>\mathrm{Mg}>\mathrm{S}>\mathrm{P}=\mathrm{K}$ \\
19 & 30 & -14 & 9 & -8 & -12 & 23 & 2 & 11 & $\mathrm{~N}>\mathrm{Ca}>\mathrm{K}>\mathrm{S}>\mathrm{P}>\mathrm{Mg}$ \\
20 & 15 & -23 & 1 & 30 & -9 & 18 & -17 & 16 & $\mathrm{~N}>\mathrm{S}>\mathrm{Ca}>\mathrm{P}>\mathrm{Mg}>\mathrm{K}$ \\
\hline
\end{tabular}

TABELA 5 - Produtividade, Índices DRIS, Índices de Balanço Nutricional (IBN) e seqüência de deficiência a excesso nutricional para os vinhedos amostrados considerando pecíolo $-1^{\mathrm{a}}$ coleta (Florescimento). São Miguel Arcanjo-SP.

\begin{tabular}{ccrrrrrrrr}
\hline Vinhedo & $\begin{array}{c}\text { Prod. } \\
\text { t/ha }\end{array}$ & \multicolumn{1}{c}{ Índice DRIS } & IBN & $\begin{array}{c}\text { Seqüência deficiência a } \\
\text { excesso }\end{array}$ \\
\hline \multicolumn{1}{c}{} & $\mathrm{N}$ & $\mathrm{P}$ & $\mathrm{K}$ & $\mathrm{Ca}$ & $\mathrm{Mg}$ & $\mathrm{S}$ & \\
\hline 01 & 30 & -9 & 3 & -25 & 9 & 5 & 17 & 11 & $\mathrm{~K}>\mathrm{N}>\mathrm{P}>\mathrm{Mg}>\mathrm{Ca}>\mathrm{S}$ \\
02 & 27 & -44 & 10 & 31 & -23 & 1 & 24 & 22 & $\mathrm{~N}>\mathrm{Ca}>\mathrm{Mg}>\mathrm{P}>\mathrm{S}>\mathrm{K}$ \\
03 & 18 & -34 & -13 & 9 & 9 & 13 & 17 & 16 & $\mathrm{~N}>\mathrm{P}>\mathrm{K}=\mathrm{Ca}>\mathrm{Mg}>\mathrm{S}$ \\
04 & 40 & -3 & 3 & 2 & 5 & -5 & -2 & 3 & $\mathrm{Mg}>\mathrm{N}>\mathrm{S}>\mathrm{K}>\mathrm{P}>\mathrm{Ca}$ \\
05 & 32 & -6 & -9 & -1 & 22 & -16 & 10 & 11 & $\mathrm{Mg}>\mathrm{P}>\mathrm{N}>\mathrm{K}>\mathrm{S}>\mathrm{Ca}$ \\
06 & 41 & 15 & -39 & 47 & 35 & -81 & 22 & 40 & $\mathrm{Mg}>\mathrm{P}>\mathrm{N}>\mathrm{S}>\mathrm{Ca}>\mathrm{K}$ \\
07 & 38 & -17 & -14 & -28 & 5 & 12 & 43 & 20 & $\mathrm{~K}>\mathrm{N}>\mathrm{P}>\mathrm{Ca}>\mathrm{Mg}>\mathrm{S}$ \\
08 & 29 & -5 & -9 & 31 & 4 & 12 & -32 & 16 & $\mathrm{~S}>\mathrm{P}>\mathrm{N}>\mathrm{Ca}>\mathrm{Mg}>\mathrm{K}$ \\
09 & 18 & -3 & 3 & 1 & 14 & -11 & -4 & 6 & $\mathrm{Mg}>\mathrm{S}>\mathrm{N}>\mathrm{K}>\mathrm{P}>\mathrm{Ca}$ \\
10 & 37 & -17 & 8 & 6 & 1 & 5 & -2 & 6 & $\mathrm{~N}>\mathrm{S}>\mathrm{Ca}>\mathrm{Mg}>\mathrm{K}>\mathrm{P}$ \\
11 & 33 & -24 & -45 & 10 & 5 & 46 & 9 & 23 & $\mathrm{P}>\mathrm{N}>\mathrm{Ca}>\mathrm{S}>\mathrm{K}>\mathrm{Mg}$ \\
12 & 18 & -8 & -4 & 9 & -3 & -1 & 7 & 5 & $\mathrm{~N}>\mathrm{P}>\mathrm{Ca}>\mathrm{Mg}>\mathrm{S}>\mathrm{K}$ \\
13 & 27 & 18 & -4 & 5 & 13 & -28 & -4 & 12 & $\mathrm{Mg}>\mathrm{P}>\mathrm{S}>\mathrm{K}>\mathrm{Ca}>\mathrm{N}$ \\
14 & 25 & 17 & 2 & -14 & 9 & -26 & 12 & 13 & $\mathrm{Mg}>\mathrm{K}>\mathrm{P}>\mathrm{Ca}>\mathrm{S}>\mathrm{N}$ \\
15 & 23 & 0 & -8 & 3 & -3 & 0 & 8 & 4 & $\mathrm{P}>\mathrm{Ca}>\mathrm{N}=\mathrm{Mg}>\mathrm{K}>\mathrm{S}$ \\
16 & 38 & -20 & -6 & 6 & -11 & 18 & 13 & 12 & $\mathrm{~N}>\mathrm{Ca}>\mathrm{P}>\mathrm{K}>\mathrm{S}>\mathrm{Mg}$ \\
17 & 30 & 10 & 13 & -18 & -2 & 10 & -13 & 11 & $\mathrm{~K}>\mathrm{S}>\mathrm{Ca}>\mathrm{N}>\mathrm{Mg}>\mathrm{P}$ \\
18 & 21 & 4 & 12 & -34 & -11 & 33 & -4 & 16 & $\mathrm{~K}>\mathrm{Ca}>\mathrm{S}>\mathrm{N}>\mathrm{P}>\mathrm{Mg}$ \\
19 & 30 & -10 & 1 & 6 & -21 & 8 & 16 & 10 & $\mathrm{Ca}>\mathrm{N}>\mathrm{P}>\mathrm{K}>\mathrm{Mg}>\mathrm{S}$ \\
20 & 15 & -22 & -38 & 17 & -7 & 0 & 50 & 22 & $\mathrm{P}>\mathrm{N}>\mathrm{Ca}>\mathrm{Mg}>\mathrm{K}>\mathrm{S}$ \\
\hline
\end{tabular}

TABELA 6 - Produtividade, Índices DRIS, Índices de Balanço Nutricional (IBN) e seqüência de deficiência a excesso nutricional para os vinhedos amostrados considerando pecíolo $-2^{\mathrm{a}}$ coleta (Fruto entre ervilha e meia-baga). São Miguel Arcanjo-SP.

\begin{tabular}{ccrrrrrrrr}
\hline Vinhedo & $\begin{array}{c}\text { Prod. } \\
\text { t/ha }\end{array}$ & \multicolumn{1}{c}{ Índice DRIS } & IBN & $\begin{array}{c}\text { Seqüência deficiência a } \\
\text { excesso }\end{array}$ \\
\hline \multicolumn{1}{c}{} & $\mathrm{N}$ & $\mathrm{P}$ & $\mathrm{K}$ & $\mathrm{Ca}$ & $\mathrm{Mg}$ & $\mathrm{S}$ & \\
\hline 01 & 30 & -26 & 13 & -12 & 4 & 8 & 12 & 12 & $\mathrm{~N}>\mathrm{K}>\mathrm{Ca}>\mathrm{Mg}>\mathrm{S}>\mathrm{P}$ \\
02 & 27 & -32 & 19 & 0 & -17 & 1 & 28 & 16 & $\mathrm{~N}>\mathrm{Ca}>\mathrm{K}>\mathrm{Mg}>\mathrm{P}>\mathrm{S}$ \\
03 & 18 & -36 & 6 & -5 & -7 & 30 & 11 & 16 & $\mathrm{~N}>\mathrm{Ca}>\mathrm{K}>\mathrm{P}>\mathrm{S}>\mathrm{Mg}$ \\
04 & 40 & 32 & -32 & -18 & 26 & -28 & 20 & 26 & $\mathrm{P}>\mathrm{Mg}>\mathrm{K}>\mathrm{S}>\mathrm{Ca}>\mathrm{N}$ \\
05 & 32 & -3 & -5 & -10 & 22 & -13 & 9 & 10 & $\mathrm{Mg}>\mathrm{K}>\mathrm{P}>\mathrm{N}>\mathrm{S}>\mathrm{Ca}$ \\
06 & 41 & -5 & -41 & 15 & 28 & -25 & 29 & 24 & $\mathrm{P}>\mathrm{Mg}>\mathrm{N}>\mathrm{K}>\mathrm{Ca}>\mathrm{S}$ \\
07 & 38 & -9 & 1 & 4 & -2 & -13 & 19 & 8 & $\mathrm{Mg}>\mathrm{N}>\mathrm{Ca}>\mathrm{P}>\mathrm{K}>\mathrm{S}$ \\
08 & 29 & 16 & -50 & -4 & 7 & 11 & 20 & 18 & $\mathrm{P}>\mathrm{K}>\mathrm{Ca}>\mathrm{Mg}>\mathrm{N}>\mathrm{S}$ \\
09 & 18 & 10 & -7 & 25 & 5 & -16 & -18 & 14 & $\mathrm{~S}>\mathrm{Mg}>\mathrm{P}>\mathrm{Ca}>\mathrm{N}>\mathrm{K}$ \\
10 & 37 & -20 & 29 & -16 & -2 & 15 & -5 & 14 & $\mathrm{~N}>\mathrm{K}>\mathrm{S}>\mathrm{Ca}>\mathrm{Mg}>\mathrm{P}$ \\
11 & 33 & 1 & -20 & -4 & -11 & 31 & 2 & 12 & $\mathrm{P}>\mathrm{Ca}>\mathrm{K}>\mathrm{N}>\mathrm{S}>\mathrm{Mg}$ \\
12 & 18 & -21 & -8 & 2 & -2 & 27 & 2 & 10 & $\mathrm{~N}>\mathrm{P}>\mathrm{Ca}>\mathrm{K}=\mathrm{S}>\mathrm{Mg}$ \\
13 & 27 & -10 & -6 & 8 & 2 & -7 & 13 & 8 & $\mathrm{~N}>\mathrm{Mg}>\mathrm{P}>\mathrm{Ca}>\mathrm{K}>\mathrm{S}$ \\
14 & 25 & 6 & -12 & 14 & 1 & -31 & 22 & 14 & $\mathrm{Mg}>\mathrm{P}>\mathrm{Ca}>\mathrm{N}>\mathrm{K}>\mathrm{S}$ \\
15 & 23 & -16 & 2 & 13 & 2 & 10 & -11 & 9 & $\mathrm{~N}>\mathrm{S}>\mathrm{P}>\mathrm{Ca}>\mathrm{Mg}>\mathrm{K}$ \\
16 & 38 & 17 & -42 & -34 & 6 & 44 & 10 & 26 & $\mathrm{P}>\mathrm{K}>\mathrm{Ca}>\mathrm{S}>\mathrm{N}>\mathrm{Mg}$ \\
17 & 30 & 13 & 6 & 5 & -7 & -15 & -2 & 8 & $\mathrm{Mg}>\mathrm{Ca}>\mathrm{S}>\mathrm{K}>\mathrm{P}>\mathrm{N}$ \\
18 & 21 & 10 & 23 & 10 & -6 & 8 & -45 & 17 & $\mathrm{~S}>\mathrm{Ca}>\mathrm{Mg}>\mathrm{N}=\mathrm{K}>\mathrm{P}$ \\
19 & 30 & 2 & -2 & -30 & 9 & 18 & 4 & 11 & $\mathrm{~K}>\mathrm{P}>\mathrm{N}>\mathrm{S}>\mathrm{Ca}>\mathrm{Mg}$ \\
20 & 15 & 34 & -96 & 9 & 35 & 30 & -12 & 36 & $\mathrm{P}>\mathrm{S}>\mathrm{K}>\mathrm{Mg}>\mathrm{N}>\mathrm{Ca}$ \\
\hline
\end{tabular}

TABELA 7 - Produtividade, Índices DRIS, Índices de Balanço Nutricional (IBN) e seqüência de deficiência a excesso nutricional para os vinhedos amostrados considerando pecíolo $-3^{\mathrm{a}}$ coleta (Início de maturação dos frutos).

\begin{tabular}{ccrrrrrrrr}
\hline Vinhedo & $\begin{array}{c}\text { Prod. } \\
\text { t/ha }\end{array}$ & \multicolumn{1}{c}{ Índice DRIS } & & IBN & $\begin{array}{c}\text { Seqüência deficiência a } \\
\text { excesso }\end{array}$ \\
\hline \multicolumn{1}{c}{} & $\mathrm{N}$ & $\mathrm{P}$ & $\mathrm{K}$ & $\mathrm{Ca}$ & $\mathrm{Mg}$ & $\mathrm{S}$ & \\
\hline 01 & 30 & -17 & 4 & -40 & 32 & 15 & 6 & 19 & $\mathrm{~K}>\mathrm{N}>\mathrm{P}>\mathrm{S}>\mathrm{Mg}>\mathrm{Ca}$ \\
02 & 27 & -24 & 21 & -2 & -7 & -2 & 14 & 12 & $\mathrm{~N}>\mathrm{Ca}>\mathrm{K}=\mathrm{Mg}>\mathrm{S}>\mathrm{P}$ \\
03 & 18 & -6 & 12 & -12 & 0 & 16 & -10 & 9 & $\mathrm{~K}>\mathrm{S}>\mathrm{N}>\mathrm{Ca}>\mathrm{P}>\mathrm{Mg}$ \\
04 & 40 & -2 & -11 & -1 & 15 & -15 & 15 & 10 & $\mathrm{Mg}>\mathrm{P}>\mathrm{N}>\mathrm{K}>\mathrm{Ca}=\mathrm{S}$ \\
05 & 32 & -6 & -4 & -18 & 34 & -8 & 1 & 12 & $\mathrm{~K}>\mathrm{Mg}>\mathrm{N}>\mathrm{P}>\mathrm{S}>\mathrm{Ca}$ \\
06 & 41 & -3 & -42 & -9 & 38 & -15 & 31 & 23 & $\mathrm{P}>\mathrm{Mg}>\mathrm{K}>\mathrm{N}>\mathrm{S}>\mathrm{Ca}$ \\
07 & 38 & 11 & -6 & 11 & -1 & -4 & -12 & 8 & $\mathrm{~S}>\mathrm{P}>\mathrm{Mg}>\mathrm{Ca}>\mathrm{N}=\mathrm{K}$ \\
08 & 29 & -11 & -36 & 5 & 4 & 27 & 11 & 16 & $\mathrm{P}>\mathrm{N}>\mathrm{Ca}>\mathrm{K}>\mathrm{S}>\mathrm{Mg}$ \\
09 & 18 & -6 & -12 & 33 & 1 & -8 & -8 & 11 & $\mathrm{P}>\mathrm{Mg}=\mathrm{S}>\mathrm{N}>\mathrm{Ca}>\mathrm{K}$ \\
10 & 37 & 2 & 33 & -28 & 10 & 17 & -34 & 21 & $\mathrm{~S}>\mathrm{K}>\mathrm{N}>\mathrm{Ca}>\mathrm{Mg}>\mathrm{P}$ \\
11 & 33 & 8 & -21 & -6 & -8 & 20 & 7 & 12 & $\mathrm{P}>\mathrm{Ca}>\mathrm{K}>\mathrm{S}>\mathrm{N}>\mathrm{Mg}$ \\
12 & 18 & -7 & -1 & -9 & -2 & 10 & 9 & 6 & $\mathrm{~K}>\mathrm{N}>\mathrm{C}>\mathrm{P}>\mathrm{S}>\mathrm{Mg}$ \\
13 & 27 & 15 & -30 & -7 & -1 & -16 & 38 & 18 & $\mathrm{P}>\mathrm{Mg}>\mathrm{K}>\mathrm{Ca}>\mathrm{N}>\mathrm{S}$ \\
14 & 25 & 2 & -9 & 7 & 4 & -29 & 24 & 12 & $\mathrm{Mg}>\mathrm{P}>\mathrm{N}>\mathrm{Ca}>\mathrm{K}>\mathrm{S}$ \\
15 & 23 & 2 & 1 & 4 & -6 & 4 & -4 & 4 & $\mathrm{Ca}>\mathrm{S}>\mathrm{P}>\mathrm{N}>\mathrm{K}=\mathrm{Mg}$ \\
16 & 38 & -12 & -20 & -3 & 0 & 45 & -10 & 15 & $\mathrm{P}>\mathrm{N}>\mathrm{S}>\mathrm{K}>\mathrm{Ca}>\mathrm{Mg}$ \\
17 & 30 & 12 & -3 & 27 & -6 & -10 & -20 & 13 & $\mathrm{~S}>\mathrm{Mg}>\mathrm{Ca}>\mathrm{P}>\mathrm{N}>\mathrm{K}$ \\
18 & 21 & 4 & 26 & 11 & -14 & -2 & -25 & 14 & $\mathrm{~S}>\mathrm{Ca}>\mathrm{Mg}>\mathrm{N}>\mathrm{K}>\mathrm{P}$ \\
19 & 30 & -20 & 18 & 1 & -14 & 22 & -7 & 14 & $\mathrm{~N}>\mathrm{Ca}>\mathrm{S}>\mathrm{K}>\mathrm{P}>\mathrm{Mg}$ \\
20 & 15 & -13 & -29 & 28 & 7 & 24 & -16 & 20 & $\mathrm{P}>\mathrm{S}>\mathrm{N}>\mathrm{Ca}>\mathrm{Mg}>\mathrm{K}$ \\
\hline
\end{tabular}


TABELA 8 - Correlação entre IBN e produtividade, considerandose os órgãos e as épocas de coleta da folha. São Miguel Arcanjo-SP.

\begin{tabular}{ccc}
\hline Órgão da folha & Épocas de Coleta & Macronutrientes \\
\hline \multirow{2}{*}{ Limbo } & Florescimento & $-0,1637$ \\
& Ervilha/meia-baga & 0,2906 \\
& Maturação & 0,0925 \\
& & \\
\multirow{2}{*}{ Pecíolo } & Florescimento & 0,2063 \\
& Ervilha/meia-baga & 0,0156 \\
& Maturação & 0,0862 \\
\hline
\end{tabular}

\section{REFERÊNCIAS}

BATAGLIA,O.C. DRIS-Citros-Uma alternativa para avaliar a nutrição das plantas. Laranja, Cordeirópolis, v.10, n.2, p.565576,1989 .

BATAGLIA,O.C.; DECHEN,A.R. Critérios alternativos para diagnose foliar. In: SIMPÓSIO AVANÇADO DE QUÍMICA E FERTILIDADE DO SOLO, 1., 1986. Piracicaba. Anais...Campinas: Fundação Cargill, 1986. p.115-136.

BEATTIE, J.M.; FORSHEY, C.G. A survey of the nutrient element status of Concord grapes in Ohio. Proceedings of the American Society Horticultural Science, Alexandria, n.64, p.21-28, 1954.

BEAUFILS, E.R. Diagnosis and Recommendation Integrated System (DRIS). A general scheme for experimentation and calibration based on principles developed from research in plant nutrition. Pietermaritzbarg, South Africa: University of Natal. 1973. 132p. Soil Science (Bulletin, 1).

BEAUFILS, E.R. Recherche d'une exploration rationelle de l'Hevea basée sur l'analyse mineral de diverses parties de la plante. In: CONGRESS SOIL SCIENCE, 7., 1956. Paris. Proceedings. S.1., s.ed., 1956. p. 360-374.

CHRISTENSEN, L.P.; KASIMATIS, A.N.; JENSEN, E.L.. Grapevine nutrition and fertilization in San Joaquin Valley. Berkeley: University of California, 1982. 40p. (Publication, 4087).

ESCANO, C.R.; JONES, C.A.; VEHARA, G. Nutrient diagnosis in corn grow on Hydric Dystrandepts: II Comparition of two systems of tissue diagnosis. Soil Science Society American Journal, Madison, n.45, p.1140-1144, 1981.

GALLO,J.R.; OLIVEIRA,A.S. Variações sazonais na composição mineral de folhas de videira e efeitos do porta-enxerto e da presença de frutos. Bragantia, Campinas, v.19, p.883-889, 1960.

HANSON, R.G. DRIS evaluation of N, P, K status of the determinant soybeans in Brazil. Communications Soil Science Plant Analysis, New York, v.12, n.9, p.933-948, 1981.

HIROCE, R.; TERRA, M.M. Teores de macronutrientes em pecíolo e limbo da videira 'Niagara Rosada'. In: CONGRESSO
BRASILEIRO DE FRUTICULTURA, 7., 1983. Florianópolis. Anais...Florianópolis: SBF, 1984.v.4, p.1184-1187.

JONES, C.A. Proposed modifications of the diagnosis and recommendation integrated system (DRIS) for interpreting plant analyses. Communications Soil Science Plant Analysis, New York, v.12, n.9, p.785-974, 1981.

JONES, C.A; BOWEN, J.E. Comparative DRIS and crop log analysis diagnosis of sugarcane tissue analysis. Agronomy Journal, Madison, n.73, p.941-944, 1981.

JONES, M.B.; CENTER, D.M.; VAUGHN, C.E.; BELL, F.L. Using DRIS to assay nutrients in sub clover. California Agricultural, Berkeley, n.40, p.19-21, 1986.

LEITE, R.A. Avaliação do estado nutricional do cafeeiro Conilon no Estado do Espírito Santo utilizando diferentes métodos de interpretação de análise foliar. 87p. Tese de Doutorado. Universidade Federal de Viçosa, 1993.

LETZSCH, N.S.; SUMNER, M.E. Effect of population size and gield level in selection of diagnosis and recommendation integrated system (DRIS) norms. Communications in Soil Science and Plant Analysis, New York, v.15, n.9, p. 997-1.006, 1984.

MALAVOLTA, E; VITTI, G.C.; OLIVEIRA, S.A. Avaliação do estado nutricional das plantas, Potafos, Piracicaba, 1989.201p.

MEYER, J.H. Advances in the interpretation of foliar analysis of sugarcane in the South African Sugar Industry. South African Sugar Journal, Durban, v.59, n.11, p. 569-585, 1975.

SCHALLER, K. Assessment of nutrient status of grapevines by leaf analysis in four P-fertilization studies with special regard to the DRIS system. Mitteilungen Klosterneuburg rebe und Wein, Obstbau und Fruchteverwertung, v.38, n.4, p.151-163, 1988. /Resumo em Horticultural Abstracts, v.58, n.11, p.920, Nov. 1988.

SILVA, N.M.da; HIROCE, R.; FUZATTO, M.G. Efeito da adubação sobre o desenvolvimento e a produção de duas variedades paulistas de algodoeiro, em Latossol Roxo intensamente cultivado. Campinas: Instituto Agronômico, 1971. $8 \mathrm{p}$.

SNÖECK, J. Caféier. In: MARTIN-PRÉVEL, P.; GAGNARD, J.; GAUTIER, P. L'Analyse végétale dans de contrôle de l'alimentation des plantes. Paris, Techinique et DocumentationLavousier, 1984. p. 473-495.

SUMNER, M.E. A new approach for predicting nutrients needs for increase crop yield. Fertilizer Soil, Peoria, v. 22, p. 68-78, 1978. 
SUMNER, M.E. Preliminary NPK foliar diagnostic norms for wheat. Communications in Soil Science Plant Analysis, New York, v.8, n.2, p.149-167, 1977.

SUMNER, M.E. Use of the DRIS system in foliar diagnosis of crops at high yield level. Communications in Soil Science Plant Analysis, New York, v.8, n.2, p.252-268, 1977a.

SUMNER, M.E.; BEAUFILS, E.R. Diagnosis of the NPK requirements of sugarcane irrespective of plant age and season using Beaufils System (DRIS). Proceedings of South African Sugar Technologists Association, Mounth Edgecombe, (6/7): 1$5,1975$.

TERRA, M.M. Nutrição, calagem e adubação. In: POMMER, C.V. Uva: tecnologia de produção, pós-colheita, mercado. ed. por C.V.Pommer. Porto Alegre: Cinco Continentes, 2003. p. 405-476.

TERRA, M.M.; COSTA, F.; SANTOS, W.R.dos; PIRES, E.J.P. Evaluation of the nutritional condition of Italia grapes (Pirovano 65 ) at three different development phases in the region of Jundiaí, Brazil, In: INTERNATIONAL HORTICULTURALCONGRESS, 16. 2002,Toronto. Abstracts...Toronto: ISHS, 2002. p.164.

TERRA, M.M.; GUILHERME, M.A.S.; SANTOS, W.R.dos PIRES, E.J.P.; POMMER, C.V.; BOTELHO, R.V. Avaliação do estado nutricional da videira 'Itália' na região de Jales, SP, usando o sistema integrado de diagnose e recomendação. Revista Brasileira de Fruticultura, v.25, n.2, p.309-314, 2003.
TERRA, M.M.; PIRES, E.J.P.; BOTELHO, R.V. Os caminhos da viticultura paulista. Atualidades Agrícolas, São Paulo, p.10-12, abril de 2005

TERRA, M.M.; PIRES, E.J.P.; NOGUEIRA, N.A.M. Tecnologia para a produção de uva Itália na região noroeste do Estado de São Paulo, $2^{\text {a }}$ ed. Ver. atual. Campinas: Coordenadoria de Assistência Técnica Integral, 1998.81p. (Documento Técnico,97).

WALWORTH, J.L.; SUMNER, M.E. The diagnosis and recommendation integrated system. In: STEWART, B.A. Advances in Soil Science, New York: Springer - Verlag, 1987. p.150-188.

ZAMBELLO JR., E.; ORLANDO FILHO, J. Diagnosis and Recommendation Integrated System (DRIS) applied to various sugarcane tissues. In: CONGRESS OF THE INTERNATIONAL SOCIETY OF SUGARCANE TECHNOLOGISTS, 17. 1979, Manila Proceedings. Manila, The Executive Committee of the ISSCT, 1980. p. 446-457. 